Original Research Article

\title{
A prospective observational study on adverse drug reactions of iohexol contrast media in computed tomography imaging studies
}

\author{
Prashant P. Shivgunde*, Ganesh Ashok Chepuri
}

\author{
University Research \\ Department, Maharashtra \\ University of Health Sciences \\ (MUHS), Mhasrul, Vani- \\ Dindori Road, Nashik, \\ Maharashtra, India
}

Received: 14 May 2019

Accepted: 05 June 2019

\section{*Correspondence to: \\ Dr. Prashant P. Shivgunde, Email: prashantshivgunde @gmail.com}

Copyright: (C) the author(s), publisher and licensee Medip Academy. This is an openaccess article distributed under the terms of the Creative Commons Attribution NonCommercial License, which permits unrestricted noncommercial use, distribution, and reproduction in any medium, provided the original work is properly cited.

\begin{abstract}
Background: Iohexol is widely used radiocontrast media in the radiology departments for the diagnostic imaging purpose. As very few studies are carried out focusing on safety profiles of this contrast media. This study was conducted to know the profile of adverse drug reactions (ADRs) induced by Iohexol in patients who are undergoing the contrast-enhanced computed tomography (CECT) examination.

Methods: Total 106 patients from CT unit of radiology department were observed for adverse drug reactions from Iohexol contrast media in 2 months duration. Acute ADRs were recorded immediately after contrast media administration by observation and personal interviews and delayed ADRs are recorded by follow up after $24 \mathrm{hrs}$.

Results: Total 23 out of 106 patients who had undergone CT imaging investigations by Iohexol contrast media had developed ADRs. Total 29 ADRs were developed in 23 patients. Out of these, 22 ADRs were acute and 7 ADRs were delayed reactions. All acute ADRs were of "probable" category and all delayed ADRs were of "possible" category according to WHO-UMC and Naranjo's causality assessment scales. All ADRs were at Level-1 according to Modified Hartwig and Siegel severity scale. Preventability assessment of ADRs using Modified Schumock and Thornton preventability scale showed that all the ADRs were of "not preventable" class. All 29 ADRs were of TYPE U (Unclassified) as per the Wills and Brown method.

Conclusions: All reactions are physiologic and are mild in nature. No lifethreatening reactions are observed during the entire study period. Development of ADRs in female patients seen higher than male patients. So, this population requires a special attention for any serious contrast media reactions.
\end{abstract}

Keywords: Adverse drug reaction, Computed tomography, Contrast media, Iohexol, Radiology

\section{INTRODUCTION}

A rapid increase in the utilization of imaging techniques in diagnostic and therapeutic radiology during the last few years has brought about a significant increment in the use of contrast media. ${ }^{1,2}$ Employing the imaging techniques with contrast media allows us to obtain the images with greater contrast details and enhances the structure of various organs, vascular system, tissues, and muscles. ${ }^{3}$

Iohexol is the third-generation non-ionic monomer, it is much more stable, more soluble. It is widely used in the radiology departments for the diagnostic imaging purpose. Ideally, there should be no any additional side effects of 
the contrast media after administration. But adverse reactions due to contrast media do occur and can lead to the life-threatening conditions. ${ }^{1,4}$ In most cases, all reactions that will occur in patients after contrast media injection are associated with the contrast media. Typically, this assumption would be true; however, in some cases, other factors could be equally responsible for such reactions, but unfortunately, these factors are seldom discussed. ${ }^{5-7}$ Adverse reactions from contrast media may present with a diverse spectrum of symptoms and can affect the various systems of the body. Some severe reactions may lead to the life-threatening emergencies if not treated and managed properly and efficiently with the time. $^{8}$

Very few studies are carried out focusing on this aspect. The safety profiles of this contrast media have been recognized as a crucial issue. This study was carried out with the aim to examine the profile of adverse drug reactions (ADRs) induced by iohexol contrast media in patients who are undergoing the contrast-enhanced computed tomography (CECT) examination and to explore the influencing factors involved in the occurrence of ADRs. The information gathered from this study helps to update the knowledge regarding ADRs of iohexol contrast media. The findings of the present study will serve radiologists, technologists, and nurses to better perceive and treat ADRs as well as aid in improving the safety of the patients undergoing CT examination.

\section{METHODS}

The site of the study was the CT Unit of Radiology Department, Regional Referral Hospital, Nashik, Maharashtra, India. This prospective observational study was carried out for the duration of 2 months. Approval from the Institutional Ethics Committee (IEC) was taken prior to initiation of the study. For this study, patients of either sex having age 18 years or above and undergoing the CT examination with iohexol as contrast media were selected. However, those patients who unable to communicate, critically ill, required emergency treatment, and not willing to follow up were excluded.
Detail information about the study and its importance was explained to the patients coming for the CT examination by providing the patient information sheet, especially regarding the confidentiality of the patient name and data. The informed consent was obtained voluntarily from each patient before enrolling in the study. All patient-related information was collected as per case record form (CRF) after the proper conversation to assess the causality relationship between the contrast media and reaction. CRF was designed to collect data on demographics, allergy status, clinical history and findings, diagnostic results as well as undergoing treatment.

The appropriate scan was done with the contrast media (iohexol) by the staff of the CT unit under the radiologist guidance. After the CT scan, the selected patient was approached for detection of any early (acute) ADRs in CT unit within one hour. For a collection of any delayed ADRs, patients were followed up after $24 \mathrm{hrs}$ from the study day \& time by contacting them on a phone call. No intervention was made in the diagnosis, treatment, or management by the investigator and co-investigator.

After total data collection the ADRs were assessed for causality, probability, severity, seriousness, and preventability by practicing the WHO-UMC Causality Assessment Scale, Naranjo's Probability Assessment Scale, Modified Hartwig and Siegel Scale for assessment of severity of ADRs, and Modified Schumock and Thornton Scale for assessment of preventability of ADRs. ${ }^{9-12}$ The classification of ADRs was done according to Wills and Brown classification of ADRs. ${ }^{13}$ Data are presented as numbers and percentage.

\section{RESULTS}

Total 106 patients were enrolled in the study. Out of that $56(52.83 \%)$ were male and $50(47.17 \%)$ were female. Out of the total of 106 patients who had undergone for CT imaging investigation by iohexol contrast media, 23 $(21.70 \%)$ had developed one or more ADRs. However, 8 (14.29\%) male patients, and $15(30 \%)$ female patients were developed one or more ADRs (Table 1).

Table 1: Total number of patients developed ADRs.

\begin{tabular}{|llll|}
\hline Gender & No. of patients with ADRs n (\%) & No. of patients without ADRs n (\%) & Total No. of patients n (\%) \\
\hline Male & $8(14.29)$ & $48(85.71)$ & $56(100)$ \\
\hline Female & $15(30.00)$ & $35(70.00)$ & $50(100)$ \\
\hline Total & $23(21.70)$ & $83(78.30)$ & $106(100)$ \\
\hline
\end{tabular}

Patient's age group wise distribution for ADRs development showed that, a high occurrence of ADRs i.e. $30.43 \%$ in patients with age group of $41-50$ years (Table 2).
The incidence of ADRs in patients from IPD was $22.22 \%$ and in OPD was $21.65 \%$ (Table 3). Total 29 ADRs were developed in 23 patients (Figure 1). Out of these 29 ADRs, $22(75.86 \%)$ were acute and $7(24.14 \%)$ were delayed 
reactions (Table 4). Out of 106 patients, Cancer patients were 54, Suspecting Cancer patients were 20 and other patients were 32 in number. An incidence rate of ADRs in
Cancer was $22.22 \%$, Suspecting Cancer $20 \%$ and in others $21.87 \%$ (Figure 2).

Table 2: Age of patients and development of ADRs.

\begin{tabular}{|lll|}
\hline Age group of patients in years & Total number of patients $\mathbf{n}(\%)$ & Number of patients developed ADRs $\mathbf{n}(\%)$ \\
\hline $18-20$ & $1(0.94)$ & $0(0)$ \\
\hline $21-30$ & $12(11.32)$ & $2(16.66)$ \\
\hline $31-40$ & $20(18.87)$ & $5(25)$ \\
\hline $41-50$ & $23(21.70)$ & $7(30.43)$ \\
\hline $51-60$ & $16(15.09)$ & $2(12.50)$ \\
\hline $61-70$ & $26(24.53)$ & $6(23.07)$ \\
\hline $71-80$ & $7(6.60)$ & $1(14.28)$ \\
\hline $81-90$ & $1(0.94)$ & $0(0)$ \\
\hline Total & $106(100)$ & $23(21.70)$ \\
\hline
\end{tabular}

Table 3: ADRs on the basis of out-patient department (OPD) and in-patient department (IPD).

\begin{tabular}{|llll|} 
Department & $\begin{array}{l}\text { Number of patients who developed } \\
\text { ADRs n }(\%)\end{array}$ & $\begin{array}{l}\text { Number of patients who not } \\
\text { developed ADRs } \mathbf{n}(\%)\end{array}$ & $\begin{array}{l}\text { Total number of } \\
\text { patients } \mathbf{n}(\%)\end{array}$ \\
\hline OPD & $21(21.65)$ & $76(78.35)$ & $97(100)$ \\
\hline IPD & $2(22.22)$ & $7(77.78)$ & $9(100)$ \\
\hline Total & $23(21.70)$ & $83(78.30)$ & $106(100)$ \\
\hline
\end{tabular}

Table 4: Distribution of ADRs on the basis of acute and delayed reactions.

\begin{tabular}{|c|c|c|c|}
\hline Acute ADRs & $\begin{array}{l}\text { Number of patients Felt } \\
\text { ADR } n(\%)\end{array}$ & Delayed ADRs & $\begin{array}{l}\text { Number of patients Felt } \\
\text { ADR } n(\%)\end{array}$ \\
\hline Giddiness & $6(27.27)$ & Fever & $3(42.86)$ \\
\hline Feeling of warmth & $6(27.27)$ & Headache & $2(28.57)$ \\
\hline Transient metallic taste & $3(13.64)$ & Loose motions & $1(14.28)$ \\
\hline Nausea & $3(13.64)$ & Vomiting & $1(14.28)$ \\
\hline Shivering & $2(9.09)$ & \multirow{4}{*}{ Total delayed ADRs } & \multirow{4}{*}{$7(100)$} \\
\hline Headache & $1(4.54)$ & & \\
\hline Fainting & $1(4.54)$ & & \\
\hline Total Acute ADRs & $22(100)$ & & \\
\hline
\end{tabular}

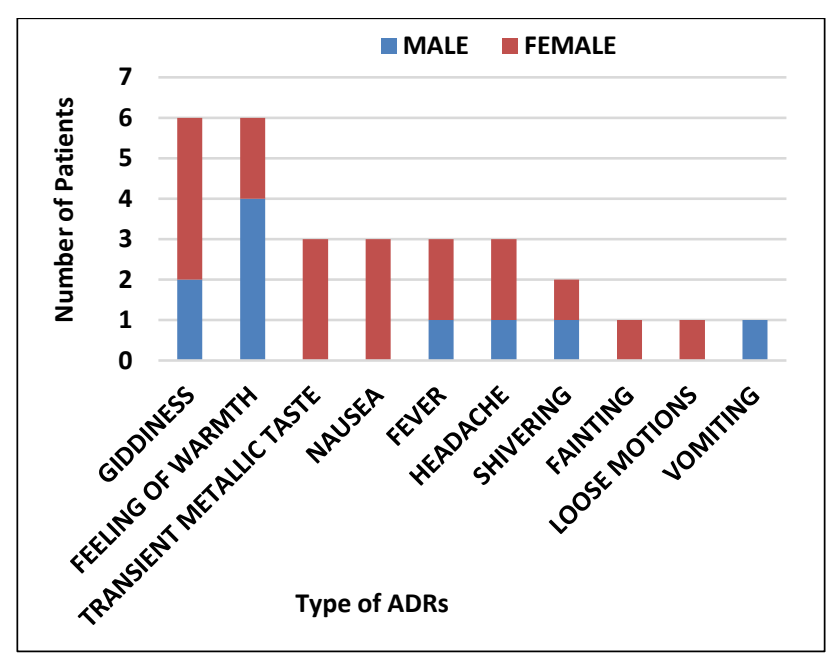

Figure 1: Type of ADRs patients developed.
WHO Causality assessment of ADRs revealed that out of 29 ADRs, $22 \quad(75.86 \%)$ ADRs (acute) were "Probably/Likely" drug-related and seven (24.14\%) ADRs (delayed) were "Possibly" drug-related. Naranjo's Causality assessment of ADRs revealed that out of 29 ADRs, 22 (75.86\%) ADRs (acute) were rated as "Probable" and 7 (24.14\%) ADRs (delayed) were rated as "Possible".

The Severity assessment of ADRs with Modified Hartwig and Siegel scale showed that all 29 (100\%) ADRs were of "Mild with Level 1".

No severe ADRs were observed. Preventability Assessment of ADRs done by Modified Schumock and Thornton scale showed that all 29 (100\%) ADRs were "Not preventable". 
Classification of ADRs by Wills and Brown method showed that all $29(100 \%)$ ADRs were of TYPE U (Unclassified) because their mechanism is not understood, and these must remain unclassified until more is known about them.

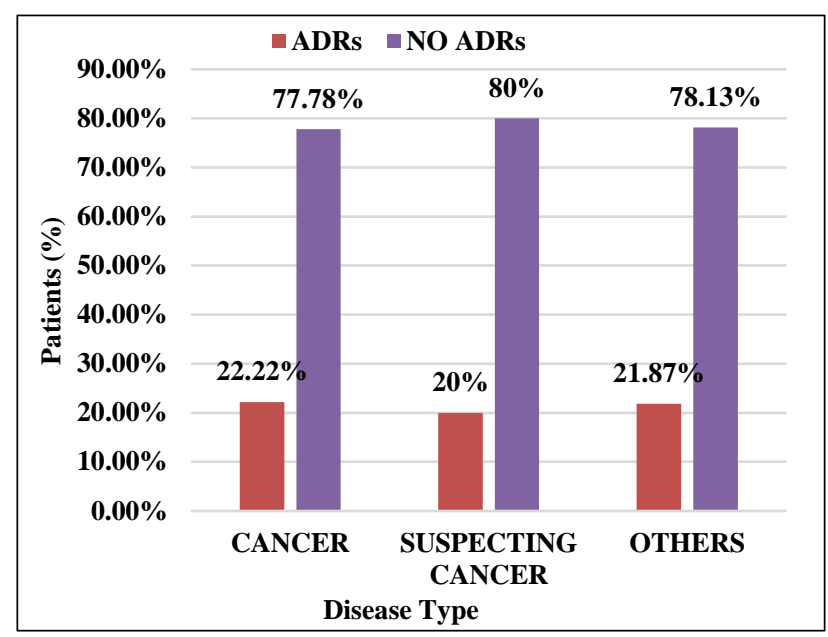

Figure 2: Incidence rate of ADRs in total 106 patients with cancer and other diseases.

\section{DISCUSSION}

In this prospective study, we have focused on the adverse drug reactions of the iohexol contrast media. Iohexol is a non-ionic contrast media and it is a commonly used diagnostic agent. Non-ionic contrast media has largely replaced the ionic contrast media. One of the reasons is that the non-ionic contrast media significantly reduce the frequency of severe and potentially life-threatening ADRs and low-osmolar iodinated agents are generally well tolerated. ${ }^{14}$ In this study, out of 106 patients, $23(21.70 \%)$ patients had developed 29 ADRs and all are of general reactions and none of them had organ-specific toxicity. The general adverse reactions are further sub-classified into acute and delayed reactions. Acute reactions usually occur within 1 hour of administration of contrast media while delayed adverse reactions usually occur from 1 hour to 1 week after contrast administration. ${ }^{15-17}$

In the present study, patients developed both acute and delayed reactions. Out of 29 ADRs, 22 (75.86\%) were acute and $7(24.14 \%)$ were delayed reactions. Acute reactions are recorded immediately after the contrast injection and the delayed reactions are recorded after 24 hours. The ADRs were in the form of giddiness, feeling of warmth, transient metallic taste, nausea, fever, shivering, headache, fainting, loose motions, and vomiting. The exact mechanism of these reactions is unclear.

Adverse reactions are categorized as either allergic-like or physiologic and organized into three general categories of severity (mild, moderate and severe). All the adverse reactions developed in this study are physiologic and mild in severity. Mild reactions are of short duration, selflimiting and generally do not require treatment. Moderate and severe reactions represent a serious degree of reactions that need immediate management and treatment. ${ }^{4}$

In this study the incidence rate of ADRs due to iohexol contrast media is $21.70 \%$, this suggests that a high rate of ADRs development. In Japan, Katayama $\mathrm{H}$ et al, had conducted a large prospective study on the safety of ionic and non-ionic contrast media in 337,647 patients and this study has shown a prevalence of adverse reactions with ionic contrast media was $12.66 \%$ in comparison with the use of non-ionic contrast media which was reduced to $3.33 \% .{ }^{14}$ The reasons for this difference in the incidence could be an inclusion of small sample size, genetic makeup, different geographical distribution, underlying diseases or contrast media used. In this study, we focused on recording even mild ADRs.

The study carried out by Namasivayam $\mathrm{S}$ et al, had reported that acute adverse reactions due to radiocontrast media are less frequent over 50 years of age and tend to be more severe in the elderly. ${ }^{15}$ However, in this study, the ADRs are most commonly seen in patients with age group of 41-50 years and followed by age group of 31-40 and then 61-70 years. In this study, the frequency of development of adverse drug reactions was higher in female patients $(30 \%)$ than male patients $(14.29 \%)$. Here a significant gender difference is seen in the development of ADRs. Both OPD and IPD patients were included in the study and the incidence rate of ADRs was not significantly differed in both the cases.

In the study of Namasivayam $\mathrm{S}$ et al, stated that the risk of acute adverse reactions to contrast media increases due to several predisposing factors such as history of previous adverse reaction to contrast media, history of allergy, asthma, dehydration, heart diseases, pre-existing renal disease, hematological conditions like sickle cell anemia, polycythemia and multiple myeloma, beta-blocker therapy, interleukin-2 therapy and non-steroidal antiinflammatory drugs. ${ }^{15}$ Histamine release is increased in patients with malignant tumors, possibly there may be a greater chance of development of anaphylactic reactions due to contrast media. ${ }^{18}$ However, our study showed not much distinction in the incidence of ADRs in patients with cancer $(22.22 \%)$, suspecting cancer $(20 \%)$ and in others $(21.87 \%)$.

Causality assessment was performed by using both WHOUMC and Naranjo's scale and results were same by both the methods that all the acute ADRs were of "probable" category and all delayed ADRs were of "possible" category. ${ }^{9,10}$ Severity assessment was performed by using Modified Hartwig and Siegel severity scale and it showed that all the ADRs were of "LEVEL 1" requiring no administration of the antidote or other drugs for treatment. ${ }_{11}$ There were no any serious reactions observed during the entire study duration. Preventability of ADRs was assessed by using Modified Schumock and Thronton preventability scale and it showed that all the ADRs were of "not preventable" class. ${ }^{12}$ All reactions were of mild in 
nature and are self-limited. Classification of ADRs was performed by using Wills and Brown method which showed that all 29 ADRs were of TYPE U (Unclassified). ${ }^{13}$

Non-ionic contrast (iohexol) media are safe drugs, most of the reactions are mild in nature. But serious reactions are also reported in the past. ${ }^{19}$ Therefore CT-unit of radiology department should be well-equipped with medications to deal with them. The limitations of the study are relatively small sample size and short duration. A re-challenge test was not done due to considering patient safety and ethical issues. Further studies are required to elaborate various aspects of ADRs due to Iohexol (non-ionic) contrast media; such as studies in different types of race and comparison studies with other contrast media for ADRs and cost-effectiveness.

\section{CONCLUSION}

Most common ADRs associated with iohexol contrast media are giddiness, feeling of warmth, transient metallic taste, nausea, fever, shivering, headache, fainting, loose motions, and vomiting. All reactions are physiologic and are mild in nature. There were no any life-threatening reactions are observed during the entire study period. Development of ADRs in female patients seen higher than male patients. So, this population requires a special attention for any serious contrast media reactions. The incidence rate of ADRs developed in this study is greater in comparison to other studies and rate of development of acute reactions is seen higher than delayed reactions. Rarely life-threatening anaphylaxis and acute hypersensitivity reactions may also occur. So, monitoring and attention towards the patients who are at high risk of developing reactions could reduce the impact of ADRs and improve the quality of care. Proper pre-procedure patient evaluation, procedure selection, and adequate prophylactic measures in serious cases should be employed to prevent adverse reactions from Iohexol contrast media.

\section{ACKNOWLEDGEMENTS}

Authors would like to thank Radiology Department, Regional Referral Hospital, Nashik for permitting to conduct the study.

Funding: No funding sources Conflict of interest: None declared

Ethical approval: The study was approved by the Institutional Ethics Committee

\section{REFERENCES}

1. Beckett KR, Moriarity AK, Langer JM. Safe use of contrast media: what the radiologist needs to know. Radio Graphics. 2015;35(6):1738-50.

2. OECD Statistics. Health, Health care utilization: Diagnostic exams. Available at: https://stats.oecd.org/
3. Contrast Enhancement Agents. In: Whitley AS, Alsop CW, Moore AD, Eds. Clark's Special Procedures in Diagnostic Imaging, 1st ed. Oxford: ButterworthHeinemann; 1999: 41-62.

4. ACR manual on contrast media: version 10.3. ACR Committee on Drugs and Contrast Media. American College of Radiology. Published 2017. Available at: https://www.acr.org/-/media/ACR/Files/ClinicalResources/Contrast_Media.pdf

5. Böhm I, Alfke H, Klose KJ. Hypersensitivity reactions and contrast medium injection: are they always related?. Eur J Radiol. 2011;80(2):368-72.

6. Böhm I. Latex allergy in patients suspected for contrast media hypersensitivity: A neglected differential diagnosis. Acta Radiol. 2010;51(6):70910.

7. Dewachter P, Laroche D, Mouton-Faivre C, BlochMorot E, Cercueil JP, Metge L, et al. Immediate reactions following iodinated contrast media injection: a study of 38 cases. Eur J Radiol. 2011;77(3):495-501.

8. Iyer RS, Schopp JG, Swanson JO, Thapa MM, Phillips GS. Safety Essentials: Acute reactions to iodinated contrast media. Can Assoc Radiol J. 2013;64(3):1939.

9. WHO. The use of the WHO-UMC system for standardised case causality assessment, Sweden. The Uppsala Monitoring Centre. Available at: http://www.who.int/medicines/areas/quality_safety/sa fety_efficacy/WHOcausality_assessment.pdf.

10. Naranjo CA, Busto U, Sellers EM, Sandor P, Ruiz I, Roberts EA, et al. A method for estimating the probability of adverse drug reactions. Clin Pharmacol Ther. 1981;30(2):239-45.

11. Hartwig SC, Siegel J, Schneider PJ. Preventability and severity in reporting adverse drug reactions. Am J Hosp Pharm. 1992;49(9):2229-32.

12. Schumock GT, Thornton JP. Focusing on the preventability of adverse drug reactions. Am J Hosp Pharm. 1992;27(6):538.

13. Wills S, Brown DA. A proposed new means of classifying adverse drug reactions to medicines. Pharm J. 1999;262(7030):163-5.

14. Katayama H, Yamaguchi K, Kozuka T, Takashima T, Seez P, Matsuura K. Adverse reactions to ionic and nonionic contrast media. a report from the Japanese committee on the safety of contrast media. Radiology. 1990;175(3):621-8.

15. Namasivayam S, Kalra MK, Torres WE, Small WC. Adverse reactions to intravenous iodinated contrast media: an update. Curr Probl Diagn Radiol. 2006;35(4):164-9.

16. Thomsen HS, Morcos SK. Contrast media safety committee of european society of urogenital radiology. Management of acute adverse reactions to contrast media. Eur Radiol. 2004;14(3):476-81.

17. Webb JA, Stacul F, Thomsen HS, Morcos SK. Members of the contrast media safety committee of the european society of urogenital radiology. late adverse reactions to intravascular iodinated contrast media. Eur Radiol. 2003;13(1):181-4. 
18. Celik I, Hoppe M, Lorenz W, Sitter H, Ishaque N, Jungraithmayr W, et al. Randomised study comparing a non-ionic with an ionic contrast medium in patients with malignancies: First answer with a new diagnostic approach. Inflamm Res. 1999;48(1):47-8.

19. Rathaur V, Dutt HK, Rawat V, Joshi P. Three cases of iohexol induced seizures in paediatric patients undergoing CECT head and the role of pharmacovigilance. J App Pharm Sci. 2014;4(8):0756.

Cite this article as: Shivgunde PP, Chepuri GA. A prospective observational study on adverse drug reactions of iohexol contrast media in computed tomography imaging studies. Int J Basic Clin Pharmacol 2019;8:1667-72. 